\title{
Design of Distributed Spiral Resonators for the Decoupling of MRI Double-Tuned RF Coils
}

\author{
Danilo Brizi, Student Member, IEEE, Nunzia Fontana, Member, IEEE, Filippo Costa, Member, IEEE, \\ Gianluigi Tiberi, Angelo Galante, Marcello Alecci and Agostino Monorchio, Fellow, IEEE
}

\begin{abstract}
Objective: A systematic analytical approach to design Spiral Resonators (SRs), acting as distributed magnetic traps (DMTs), for the decoupling of Double-Tuned (DT) RF coils suitable for Ultra-High Field (7 T) MRI is presented. Methods: The design is based on small planar SRs placed in between the two RF loops (used for signal detection of the two nuclei of interest). We developed a general framework based on a fully analytical approach to estimate the mutual coupling between the RF coils and to provide design guidelines for the geometry and number of SRs to be employed. Starting from the full-analytical estimations of the SRs geometry, electromagnetic simulations for improving and validating the performance can be carried out. Results and Conclusion: We applied the method to a test case of a DT RF coil consisting of two concentric and coplanar loops used for $7 \mathrm{~T}$ MRI, tuned at the Larmor frequencies of the proton $\left({ }^{1} \mathrm{H}\right.$, $298 \mathrm{MHz})$ and sodium $\left({ }^{23} \mathrm{Na}, 79 \mathrm{MHz}\right)$ nuclei, respectively. We performed numerical simulations and experimental measurements on fabricated prototypes, which both demonstrated the effectiveness of the proposed design procedure. Significance: The decoupling is achieved by printing the SRs on the same dielectric substrate of the RF coils thus allowing a drastic simplification of the fabrication procedure. It is worth noting that there are no physical connections between the decoupling SRs and the ${ }^{1} \mathrm{H} /{ }^{23} \mathrm{Na}$ RF coils, thus providing a mechanically robust experimental set-up, and improving the transceiver design with respect to other traditional decoupling techniques.
\end{abstract}

Index Terms-Decoupling; Distributed Magnetic Traps (DMTs); Double-Tuned (DT); Magnetic Resonance Imaging (MRI); mutual decoupling; Radio Frequency (RF) coil; Spiral Resonators (SRs).

\section{INTRODUCTION}

$\mathrm{I}^{\mathrm{N}}$ Magnetic Resonance Imaging (MRI) the mutual decoupling between two or more RF coils is one of the most

D. Brizi, F. Costa and A. Monorchio are with the Department of Information Engineering, University of Pisa, 56122 - Pisa, Italy (e-mail:, danilo.brizi@ing.unipi.it).

N. Fontana was with Consorzio Nazionale Interuniversitario per le Telecomunicazioni (CNIT), 56122 - Pisa, Italy, now is with Dept. of Ingegneria dell'Energia, dei Sistemi, del Territorio e delle Costruzioni (D.E.S.Te.C.), University of Pisa, 56122 - Pisa, Italy.

G. Tiberi is with the Department of Electrical and Electronic Engineering, London South Bank University, London, UK.

A. Galante and M. Alecci are with the Department of Life, Health and Environmental Sciences, University of L'Aquila, L'Aquila, Italy; Istituto Nazionale di Fisica Nucleare (INFN), Laboratori Nazionali del Gran Sasso, Assergi, L'Aquila, Italy; and CNR-SPIN, Department of Physical and Chemical Sciences, L'Aquila, Italy. challenging design tasks, both for single frequency arrays and for multiple tuned RF coils. These MRI RF coils configurations can be used to expand the acquired Field of View (FOV), to increase the Signal-to-Noise ratio (SNR), to reduce the total scan time, and also to allow the acquisition of information deriving from two different nuclei (e.g. DoubleTuned configuration) [1]-[9]. In general, inside the MRI bore, the available space suitable for the RF coils can be very limited, and a high filling factor of the RF coils with the biological sample is strongly desirable; thus, the RF coil elements need to be placed very close to each other, resulting in a high mutual coupling that significantly detunes the resonant frequency [10]. This problem leads to SNR degradation and a lowered efficiency of the RF transmit side. At the Larmor frequencies of $7 \mathrm{~T}$ MRI systems, the main contribution to mutual coupling can be still associated to the magnetic flux linkage between the RF coils, rather than to mutual capacitive coupling (significant for fields above $7 \mathrm{~T}$ [11]).

The problem of the mutual coupling in MRI applications has been extensively studied in the literature. The most common decoupling system for RF coils arrays is the surface overlapping technique [1]. Although this method is straightforward, it is not scalable when the number of RF coils increases, requiring additional means for decoupling. Moreover, it is not feasible for coplanar and concentric RF coils configurations, which are typically used for DT surface RF coil configurations.

In the case of DT RF coils, another well-established practice is the use of a lumped trap circuit inserted in the lower frequency RF coil loop [12]. This method can achieve good decoupling performances, but the Q-factor of the lower frequency RF coil worsens due to additional losses inside the lumped elements of the trap circuit [13], [14]. In addition, peaks of electric field next to the lumped elements (especially capacitors) can be produced, requiring the insertion of additional shielding mechanisms to avoid undesired hot spots in the sample tissues [15], [16]. Another interesting decoupling solution consists in an appropriate geometrical disposition of the DT coils [17] to minimize coupling, which is a very effective method when no particular constraints on the coils positioning are present.

A relatively recent decoupling method (Induced Current Elimination, ICE), based on an eigenvectors and eigenvalues approach, has been presented in [18], [19]. Thanks to this 
method, additional resonant elements (i.e. microstrip elements) interleaved between the RF coil loops can be placed, and the conditions necessary for decoupling analyzed. In particular, the currents induced in these decoupling elements can reduce the flux linkage between the MRI coils. Other works presented in the literature for decoupling purposes exploit additional reactive and resonant elements, variously interleaved between the MRI array RF coils [20][25]. Also, the use of magnetic walls [26]-[28] successfully demonstrated a reduction of mutual coupling between adjacent RF coils tuned at the same frequency $\left({ }^{1} \mathrm{H}\right)$. The basic operative principle is similar to the ICE technique, but here a high number of miniaturized resonators are employed instead of a single one.

This paper aims to present a general and systematic procedure useful to design distributed SRs used for the decoupling of coplanar and concentric DT $\left({ }^{1} \mathrm{H} /{ }^{23} \mathrm{Na}\right) \mathrm{RF}$ coils finding MRI applications at $7 \mathrm{~T}$. Our design adopts small planar SRs, placed in between the two RF coil loops, capable to produce a large RF decoupling. We are able to determine the minimum number of SRs to be employed, minimizing unnecessary resistive losses and thus, simplifying and improving previous similar literature solutions. Since there are no physical connections between the decoupling SRs and the DT RF coil elements, we obtain a mechanically robust experimental set-up, facilitating a transceiver design.

The paper is organized as follows: Section II provides the analytical method to estimate the mutual coupling between each RF elements, the procedure to choose an appropriate number of SRs to achieve the desired decoupling and the design criteria of the single SR; Section III reports the numerical simulations and experimental results about the chosen test-case. Finally, Conclusions are presented in Section IV.

\section{DECOUPLING APPROACH FORMULATION}

The decoupling method is based on the inclusion of a number of SRs, placed in close proximity of the tuned RF coils, acting as distributed magnetic traps (DMTs). One practical advantage in the adoption of the distributed SRs consists in the use of PCB technology that allows printing the SRs on the same dielectric substrate of the RF coils. This "in plane" decoupling method allows retaining the optimal geometric configuration for the RF coils without the need for additional design constraints.

A SR is a resonant circuit consisting of a $k$-turns planar spiral [26]-[28]. The shape of the spiral and the number of turns, $k$, can be designed to realize a suitable unit cell in terms of resonant frequency and Q-factor. SRs can reach high level of miniaturization (they are extremely subwavelength), thus allowing the realization of very compact units.

The approach introduced in the paper is aimed at estimating the optimal number of unit cells required for the RF coils decoupling. This is achieved through the analytical evaluation of the mutual coupling, by using a magneto-static approximation, between: (i) the RF coils; and (ii) each RF coil and a single SR. Finally, the equivalent lumped element circuit describing the overall system is analyzed.

As a test-case for our procedure, we considered a DT RF coil constituted by two concentric and coplanar square loops, tuned at $7 \mathrm{~T}$ and suitable for MRI. As a practical working example, we considered the two RF coils tuned at the resonant frequency of ${ }^{1} \mathrm{H}(298 \mathrm{MHz})$ and ${ }^{23} \mathrm{Na}(79 \mathrm{MHz})$, respectively. The larger size ${ }^{1} \mathrm{H}$ RF coil allows the acquisition of anatomical imaging, whereas the smaller ${ }^{23} \mathrm{Na} \mathrm{RF}$ coil provides information about physiological features, as for example in osteoarthritis or brain tumor detection [8], [12], [13], [29][32]. The mutual coupling, existing between the two resonant loops, produces a large increase of the resonant frequency of the ${ }^{1} \mathrm{H}$ channel, thus detuning the ${ }^{1} \mathrm{H}$ coil with a deleterious SNR loss.

The proposed method is able to reduce or null the mutual coupling between the ${ }^{1} \mathrm{H}$ and ${ }^{23} \mathrm{Na}$ resonant loops. This was done by means of both numerical simulations and experimental measurements on the workbench. The decoupling approach consists of the following steps:

A. Estimation of mutual coupling between the RF coils;

$B$. Determination of the number of SRs needed to compensate the coupling estimated at step $A$;

C. Determination of the SR geometry.

The three steps for optimizing the decoupling SRs are described in the following paragraphs.

\section{A. Magneto-static coupling estimation}

The first step of the proposed procedure requires evaluating the mutual coupling between the two RF coils. Under the magneto-static hypothesis, we apply the Biot-Savart formulation to estimate the mutual coupling between two generic RF coils [36]. Indeed, as stated before, the main contribution to mutual coupling at the MRI frequencies is the inductive one [11]. In general, the magnetic field produced by a given current path in a specific point can be expressed as:

$$
\vec{B}(\vec{r})=\frac{\mu_{0}}{4 \pi} \int \frac{I \overrightarrow{d l} \times \vec{r}}{|\vec{r}|^{3}},
$$

where $\mu_{0}(\mathrm{H} / \mathrm{m})$ is the magnetic permeability of the vacuum, $I$ (A) is the current amplitude flowing in the path, $\overrightarrow{d l}(\mathrm{~m})$ is an infinitesimal element of the current path and $\vec{r}(\mathrm{~m})$ is the vector distance between the infinitesimal element $\overrightarrow{d l}$ and a generic point of the space.

Without loss of generality, it is possible to describe the mutual coupling coefficient between the coils $i$ and $j$ as the magnetic flux per unit current through the surface of coil $j$ induced by the current flowing in coil $i$ :

$$
M_{i j}=\frac{\Phi_{i j}}{I_{i}} .
$$

In this way, given the geometrical design of the two RF coils, we can numerically set a unit current flowing in one of them and evaluate the mutual inductive coupling with the other RF coil, taking into account that $M_{i j}=M_{j i}$. 
Importantly, we can apply the above formalism to describe both the coupling between the two RF coils and also between each RF coil and a SR. In this way, as it will be described in Section II.B, we are able to fully calculate the components of the impedance matrix $Z$, characterizing the entire RF system. Here, we consider the decoupling procedure for an MRI array consisting of two resonant loops. It is worth noting that this procedure can be easily generalized for any type of array, properly choosing the number, position and electromagnetic behavior of the decoupling SRs.

\section{B. Number of spiral resonators determination}

Once estimated the mutual coupling values between the two RF coil loops (say $M_{12}$ ), it is necessary to design the decoupling SRs. As stated above, SRs can be employed to reduce or null the mutual coupling $M_{12}$. In particular, a suitable number $N$ of SRs has to be introduced in between the two RF coils. The network model represents one useful description of the RF system, consisting of the two RF coils and a single SR. The model is formulated using Kirchhoff's voltage law for each coil. In general, we assume that both RF MRI loops can be actively driven. Consequently, since the SR (say loop 3) is a passive element, we adopt voltage sources as driving functions applied to loop 1 (corresponding to the loop mostly affected by the resonant frequency increase) and to loop 2. Finally, the current in each coil is calculated as a function of self and mutual impedance between each element of the system. The lumped elements schematic of this system is shown in Fig. 1.

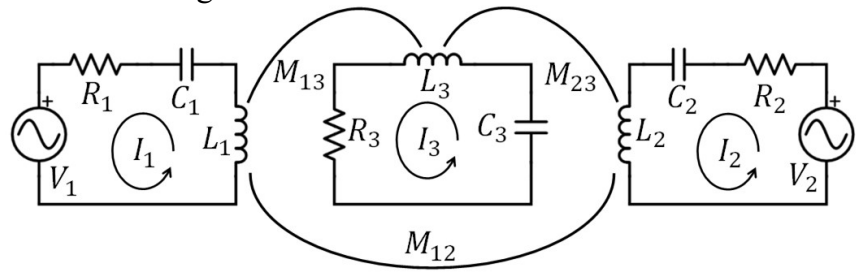

Fig. 1. Lumped elements representation of the system constituted by two fluxcoupled RF coils (numbered 1 and 2) and a decoupling SR (numbered 3). The presence of the mutual coupling between the RF coils $\left(M_{12}\right)$ and also between the RF coils and the $\mathrm{SR}\left(M_{13}, M_{23}\right)$ is highlighted.

In order to avoid unnecessary complications of the equivalent circuit model, we can hypothesize that the SRs are placed sufficiently far from each other and this, together with their reduced size, implies a negligible mutual coupling among them. Moreover, if the $N$ identical SRs are placed so that each of them interacts equally with the same RF coil, it is possible to condense the $N$ equations corresponding to each SR into a single condition. The equivalent circuit of Fig. 1 thus remains valid once we multiply the mutual terms $M_{13}, M_{23}$ and the SR self-impedance $\left(R_{3}+j \omega L_{3}-j /\left(\omega C_{3}\right)\right)$ by the factor $N$. This introduces some geometrical constraints to the positioning of the SRs, but it does not represent a strong limitation due to their small size.

We express the equations of the complete system in a matrix form, taking into account the two RF coils and the $N$ SRs. Here the SRs are considered passive elements, and this guarantees that the $\mathrm{Z}$ matrix is symmetric.

$$
\left[\begin{array}{ccc}
Z_{11} & Z_{12} & Z_{13} \\
Z_{21} & Z_{22} & Z_{23} \\
Z_{31} & Z_{32} & Z_{33}
\end{array}\right]\left[\begin{array}{c}
I_{1} \\
I_{2} \\
I_{3}
\end{array}\right]=\left[\begin{array}{c}
V_{1} \\
V_{2} \\
0
\end{array}\right]
$$

Clearly, this matrix system is a compact expression for the following three equations:

$$
\left\{\begin{array}{c}
\left(R_{1}+j \omega L_{1}-j / \omega C_{1}\right) I_{1}+j \omega M_{12} I_{2}+j \omega N M_{13} I_{3}=V_{1} \\
j \omega M_{21} I_{1}+\left(R_{2}+j \omega L_{2}-j / \omega C_{2}\right) I_{2}+j \omega N M_{23} I_{3}=V_{2} \\
j \omega N M_{31} I_{1}+j \omega N M_{32} I_{2}+N\left(R_{3}+j \omega L_{3}-j / \omega C_{3}\right) I_{3}=0
\end{array},\right.
$$

where we model the RF coils and the SRs as series resonant $R L C$ circuits with diagonal entries of the form $R+j \omega L-j /(\omega C)$.

Keeping in mind the goal of eliminating the mutual coupling between the RF coils at the resonant frequency of the (isolated) coil 1 (the ${ }^{1} \mathrm{H}$ loop, say $\omega_{01}$ ), we observe from the first equation in (4) that this condition can be achieved if $j \omega M_{12} I_{2}+j \omega N M_{13} I_{3}=0$. In previous work [12] the mutual coupling was nulled by placing a lumped parallel L-C resonant circuit (trap) in series with loop 2, while loop 1 was kept unchanged. In the present paper the same effect is obtained through at least one distributed resonant circuit (SR) inductively coupled to both coils 1 and 2, making a DMT (the meaning of "magnetic trap" will be clarified in the following).

It is convenient to rearrange the system (3) in order to highlight the cross-talking between the two RF coils (elements 1 and 2 of the equivalent circuit). To this aim, the current $I_{3}$ in the SRs can be expressed as:

$$
I_{3}=\frac{-Z_{31} I_{1}-Z_{32} I_{2}}{Z_{33}}
$$

By replacing relation (5) into the first two equations of system (3), a system explicitly describing the mutual coupling between the two RF coils in the presence of the SRs is obtained:

$$
\left[\begin{array}{cc}
Z_{11}-Z_{13} Z_{31} / Z_{33} & Z_{12}-Z_{13} Z_{32} / Z_{33} \\
Z_{21}-Z_{23} Z_{31} / Z_{33} & Z_{22}-Z_{23} Z_{32} / Z_{33}
\end{array}\right]\left[\begin{array}{l}
I_{1} \\
I_{2}
\end{array}\right]=\left[\begin{array}{l}
V_{1} \\
V_{2}
\end{array}\right] .
$$

The implementation of the decoupling method allows deriving the condition that the SRs must satisfy to decouple the RF coils. Indeed, the effective $Z_{12_{e f f}}$ parameter must be set to zero:

$$
Z_{12_{e f f}}=Z_{12}-Z_{13} Z_{32} / Z_{33}=0
$$

In the hypothesis of $N$ SRs tuned at the resonant frequency $f_{\mathrm{SR}}$ and in the absence of mutual coupling between them, this equation, at the desired resonant frequency $\omega_{01}$ of coil 1 , can be rewritten as:

$$
j \omega_{01} M_{12}=\frac{j \omega_{01} N M_{13} j \omega_{01} N M_{32}}{N\left(R_{3}+j \omega_{01} X_{3}\right)}
$$

where $X_{3}$ (equivalent inductance of the single SR self- 
impedance) is represented by:

$$
X_{3}=L_{3}-\frac{1}{\omega_{01}^{2} C_{3}} .
$$

Rearranging (8), considering that $M_{32}=M_{23}$, and distinguishing the real and the imaginary components of the right member of the equation, in order to fulfill the condition, we obtain:

$$
\left\{\begin{array}{c}
j \omega_{01} M_{12}=\frac{j \omega_{01}^{3} N M_{13} M_{23} X_{3}}{R_{3}^{2}+\omega_{01}^{2} X_{3}^{2}} \\
0=\frac{-\omega_{01}^{2} N M_{13} M_{23} R_{3}}{R_{3}^{2}+\omega_{01}^{2} X_{3}^{2}}
\end{array},\right.
$$

where the first equation of system (10) corresponds to null the imaginary component of the term $Z_{12_{e f f}}$. It must be noted that in practical conditions the second equation of (10) cannot be perfectly satisfied. This term arises because of the finite resistance of the SR. In order to keep this term low (i.e., negligible resistive losses), it is necessary that:

$$
\omega_{01}^{2} X_{3}^{2} \gg R_{3}^{2} \text {. }
$$

Under condition (11), the first equation in (10) can be satisfied when:

$$
X_{3}=\frac{N M_{13} M_{23}}{M_{12}} .
$$

By replacing (12) in (11), it is straightforward finding the minimum number $N$ of SRs such that the real part of (10) is negligible:

$$
N^{2} \gg\left(\frac{M_{12} R_{3}}{\omega_{01} M_{13} M_{23}}\right)^{2}
$$

Once a proper number of SRs is chosen, from (12) it is possible to find out the value of the reactance that the SR must have at the desired working frequency $\omega_{01}$. In this way, the imaginary component of the mutual coupling is compensated.

At this point, the real term of the mutual coupling must be evaluated; if it is not sufficiently low with the chosen $N$, then a greater number of SRs must be used in order to better verify condition (11). Indeed, the real term diminishes with an increasing number $N$ of SRs:

$$
\begin{aligned}
& \frac{-\omega^{2} N M_{13} M_{23} R_{3}}{R_{3}^{2}+\omega^{2} X_{3}^{2}} \cong \frac{-\omega^{2} N M_{13} M_{23} R_{3}}{\omega^{2} X_{3}^{2}}= \\
& =\frac{-\omega^{2} N M_{13} M_{23} R_{3}}{\omega^{2}\left(\frac{N M_{13} M_{23}}{M_{12}}\right)^{2}}=\frac{-M_{12}^{2} R_{3}}{N M_{13} M_{23}} .
\end{aligned}
$$

Obviously, the presence of the SRs has a significant effect also on the impedance of coil 1. Indeed, as shown in (6) the effective $Z_{1_{\text {eff }}}$ is written as:

$$
Z_{11_{\text {eff }}}=Z_{11}-Z_{13} Z_{31} / Z_{33} .
$$

Substituting the respective physical quantities, it becomes:

$$
\begin{aligned}
& Z_{11_{e f f}}=\left(R_{1}+\frac{\omega_{01}^{2} N M_{13} M_{31} R_{3}}{R_{3}^{2}+\omega_{01}^{2} X_{3}^{2}}\right)+ \\
& +j\left(\omega_{01} L_{1}-\frac{1}{\omega_{01} C_{1}}-\frac{\omega_{01}^{3} N M_{13} M_{31} X_{3}}{R_{3}^{2}+\omega_{01}^{2} X_{3}^{2}}\right) .
\end{aligned}
$$

This means that the employed SRs contribute with additional terms to the real and imaginary components of the self-impedance of coil 1 . Such contribute must be considered when the tuning and matching process of coil 1 is carried out. It must be noticed that the condition about the mutual coupling reduction, developed here with the described network model, is also valid in the presence of feeding ports with their own impedances; indeed, the mutual coupling is not affected by the feeding ports' properties. Moreover, the model is general and can be applied for both dual tuned and planar array configurations. Indeed, only the mutual coupling between side-by-side array elements is commonly considered significant [18]. Therefore, the decoupling scheme is the same of a dual tuned configuration, i.e. inserting opportunely spiral resonators to reduce mutual impedance between two adjacent RF coil loops.

Clearly, the presented decoupling method has a certain degree of approximation. However, we believe that the proposed analytical approach should be useful since it provides a meaningful estimation of the required number of SRs, starting only from magneto-static considerations. The alternative to this approach is a highly expensive computational simulations method, leading to an optimized solution without a physical understanding of the coupling reduction mechanism. On the contrary, in this case, the finetuning of the analytical solution can be aided through few and targeted full-wave simulations.

\section{Spiral resonator design}

The SRs, consisting of $k$-turn planar spiral loop coils, are printed on the same dielectric substrate of the RF coils.

In order to satisfy the hypothesis stated before, and act as a DMT circuit for coil 2, the SRs must fulfill the condition expressed in (11)-(13); i.e. they should present, at the resonant frequency $\omega_{01}$ of coil 1 , an appropriate reactive impedance, related to the mutual coupling coefficients and the number $N$ of SRs. The geometrical design of the single SR must be appropriate for the available space between the RF coils and also able to satisfy the decoupling criterion. Once the exploitable dimensions are determined, we can apply the following formulation in order to find the geometry and number of turns satisfying the above stated conditions.

A SR can be approximated as a $L C$ circuit [29], [30]. The total distributed inductance and capacitance can be determined starting from its geometrical parameters. In particular, we can set the dimensions $l_{x}$ and $l_{y}$, the number of turns $k$, the widths ( $w_{x}$ and $w_{y}$ ) of the conductor strip, the gap between each loop $\left(s_{x}\right.$ and $\left.s_{y}\right)$, the dielectric material $\left(\varepsilon_{r}\right.$ and $\tan \delta$ ) and its thickness $h$. Without loss of generality, we can assume for simplicity that $w_{x}=w_{y}=w$ and $s_{x}=s_{y}=s$ (Fig. 2). 


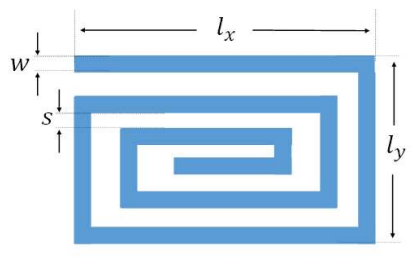

(a)

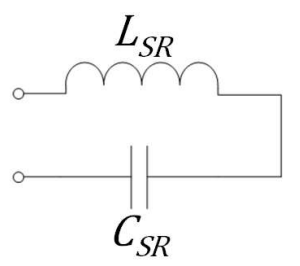

(b)
Fig. 2. Spiral resonator acting as distributed trap filter: (a) geometrical parameters (drawing not in scale); (b) magneto-static equivalent circuit (assuming no losses).

The $L_{S R}$ and $C_{S R}$ values determine the resonant frequency of the spiral:

$$
f_{S R}=\frac{1}{2 \pi \sqrt{L_{S R} C_{S R}}} .
$$

In order to estimate the distributed inductance and capacitance, we exploited a modified version of the method proposed in [29], [30], extending the formulation to rectangular spiral shapes. In particular, we modified the expression for the total strip length, with respect to the previous model presented in the literature, taking into account the two dimensions of a rectangular shape. The following expressions describe the conductor strip total length of the generic $k^{\text {th }}$ turn of a rectangular spiral:

$$
\left\{\begin{array}{l}
l_{k}=2 l_{x}-2 l_{y}-(w+s), \quad \text { if } k=1 \\
l_{k}=2\left[l_{x}-2(k-1)(w+s)\right]+ \\
+2\left[l_{y}-2(k-1)(w+s)\right], \quad \text { if } \quad k>1
\end{array} .\right.
$$

The distributed capacitance is due to adjacent branches in the spiral that realizes a capacitor. Therefore, it can be estimated starting from the total gap length of the spiral:

$$
\left\{\begin{array}{l}
l_{k}^{\text {gap }}=2\left[l_{x}+l_{y}-4(w-s)\right], \text { if } k=1 \\
l_{k}^{\text {gap }}=2\left[l_{x}-2 k \cdot w-2(k-1) s\right]+ \\
+2\left[l_{y}-2 k \cdot w-2(k-1) s\right], \text { if } k>1
\end{array} .\right.
$$

As described in [29], [30], the presence of the dielectric substrate clearly affects the resonant frequency by multiplying the total capacitance value for the effective dielectric permittivity. We used a modified expression, given in [37], to estimate the effective $\varepsilon_{r}$ :

$$
\varepsilon_{r}^{e f f}=\frac{\varepsilon_{r}+1}{2}+\left(\frac{\varepsilon_{r}+1}{2}-1\right) e^{-\left(\frac{\alpha h}{(2 w+l)}\right)} .
$$

With respect to the formulation reported in [29], [30], (20) converges, for increasing thickness $h$, to the average permittivity value between air and substrate with a speed that can be modulated by the parameter $\alpha$. This behavior is more realistic rather than a saturation value equal to the permittivity of the employed dielectric, since the substrate is present only on one side of the coil. Thanks to this formulation, we can design a SR with the desired resonant frequency, which also satisfies the geometrical constraints required by the RF coil loops configuration.
However, it was demonstrated in [38] that the lumped inductance and capacitance retrieved by using this analytical model based only on the resonator's geometrical properties are not accurate. To overcome this limit, once the spiral resonator satisfying the geometrical constraints and working frequency is designed, we characterize its effective RLC equivalent circuit by using the procedure presented in [38]. This step is fundamental to estimate accurately the reactive impedance of the resonator required for decoupling and, eventually, refining its design. Nonetheless, the condition (12) will be satisfied close to the SR's resonant frequency, slightly above or below it depending on the signs of the different mutual coupling coefficients.

At this point, we must fix the number of SRs to compensate the mutual coupling between the RF coils. As reported in Section II.A, we can evaluate the mutual coupling between each RF coil and the designed SR, by using the magneto-static approximation. Afterwards, we follow the procedure given in Section II.B; in particular, the required number $N$ of SRs is given in (13). In addition, the positioning of the $N$ SRs must be carefully evaluated. It is necessary to place the SRs as far as possible from each other, to make negligible the mutual coupling between them but keeping the same distance from the main coils to have constant mutual induction values.

\section{CASE STUDY}

As introduced in Section II, we chose as test case the design of a 7 T DT RF coil made by two coplanar and concentric rectangular loops, with the inner RF loop tuned at the Larmor frequency of ${ }^{23} \mathrm{Na}(79 \mathrm{MHz})$, whereas the outer RF loop is tuned at the Larmor frequency of ${ }^{1} \mathrm{H}(298 \mathrm{MHz})$. Because of the coplanar and concentric geometry, a strong mutual coupling arises, causing a large upshift of the ${ }^{1} \mathrm{H}$ RF coil frequency.

\section{A. Analytical design}

The ${ }^{1} \mathrm{H}$ RF coil is a rectangular loop made by a $4 \mathrm{~mm}$ width copper strip with external sizes equal to $116 \mathrm{~mm}$ and $108 \mathrm{~mm}$. The inner ${ }^{23} \mathrm{Na}$ loop measures $92 \mathrm{~mm} \times 84 \mathrm{~mm}$ (copper width of $4 \mathrm{~mm}$ ). The resulting gap size between the two RF coils is equal to $8 \mathrm{~mm}$. Given this geometrical setup, we numerically implemented the analytical procedure for the mutual coupling estimation (as described in Section II.A): the estimated magneto-static mutual coupling coefficient between the two RF coils is equal to $M_{{ }^{1}{ }_{H}{ }^{23} \mathrm{Na}}=117 \mathrm{nH}$.

Through preliminary full-wave simulations, we noticed that, although each isolated RF coil was correctly tuned at its resonant frequency, the presence of the mutual coupling caused, as expected, a relevant upshift of the ${ }^{1} \mathrm{H}$ RF coil resonant frequency (about $25 \mathrm{MHz}$ ) and a negligible downshift of the ${ }^{23} \mathrm{Na}$ RF coil resonant frequency.

To null the mutual coupling, we used 4 SRs placed symmetrically in the gap between the two RF coils, exploiting the maximum width of $8 \mathrm{~mm}$, and positioned at half-length of their branches. This geometry guarantees: (i) negligible coupling among SRs; and (ii) the mutual coupling invariance 
among each SR and the ${ }^{1} \mathrm{H}$ RF coil, as well with respect to the ${ }^{23} \mathrm{Na} \mathrm{RF}$ coil. Thus, the requisites in the analytical calculations presented in Section II are fulfilled.

By exploiting the formulation reported in Section II.C, we designed a $k=6$ turns planar SR tuned, when isolated, at about $300 \mathrm{MHz}$, with size $l_{x}=13.7 \mathrm{~mm}$ and $l_{y}=6.7 \mathrm{~mm}$. The conductor width $w$ was set equal to $0.127 \mathrm{~mm}$, with a $35 \mu \mathrm{m}$ thickness. The estimated self-inductance value was $748 \mathrm{nH}$, whereas the self-capacitance resulted in $0.37 \mathrm{pF}$ [38].

We then analytically estimated the mutual coupling between each RF coil and a single SR placed in the gap between them. Because of the chosen geometry, the mutual coupling coefficient of the SR resulted practically equivalent for both the RF coils, but with inverted sign: $M_{{ }^{1} H S R}=24 \mathrm{nH}=-M_{{ }^{23} \text { NaSR }}$ . The sign inversion is due to the symmetric position of the SRs with respect to the RF coils and the flux induced by the currents (same sign) circulating in the RF coils is opposite. In addition, we analytically verified - through the magnetostatic procedure described previously — that, in the present conditions, the mutual coupling coefficient between each pair of SRs was actually negligible (equal to $76.8 \mathrm{pH}$ ).

Now, as described in Section II.B, we must satisfy inequality (13), choosing the minimum number of SRs that guarantees the decoupling condition. The resistance value $R_{3}$ of the single SR was estimated as described in [38], resulting in $10.8 \Omega$. Choosing $N=4$, the condition in (13) was close to being satisfied (16 $\gg 1.16$, one order of magnitude). Thus, $N=4$ was the minimum number of SRs able to guarantee condition (13), allowing a symmetrical positioning of the SRs around the two coils. This has the additional beneficial effect in terms of symmetry of the magnetic field distribution inside the FOV of the DT RF coil.

Under the retrieved mutual coupling coefficients, the lumped parameters and number of employed SRs (summarized in Table I), the ideal reactance of the single SR should satisfy (12) (i.e $X_{3}=-19.7 \mathrm{nH}$ ) at the frequency of interest $(298 \mathrm{MHz})$.

In addition, it is worth noting that the 4 SRs produced a reflected impedance value for coil 1 that must be matched through an appropriate network in a practical scenario. The detailed CAD model is reported in the next section.

\section{B. Full-wave simulation}

Afterwards, we performed full-wave simulation of the system described above (CST Microwave Studio, Darmstadt). Initially, the two standalone RF coils were tuned and matched at about $298 \mathrm{MHz}$ and $79 \mathrm{MHz}$. Each RF coil consisted of a rectangular loop $\left({ }^{1} \mathrm{H}: 116 \mathrm{~mm} \times 108 \mathrm{~mm} ;{ }^{23} \mathrm{Na}: 92 \mathrm{~mm} \times 84\right.$ $\mathrm{mm}$ ) made by a $4 \mathrm{~mm}$ wide copper strip, etched on a $0.8 \mathrm{~mm}$ thick dielectric substrate (Arlon, $\left.\varepsilon_{r}=3.45, \tan \delta=0.0035\right)$ and interrupted by lumped elements (see Fig. 3).

The desired resonant frequencies of the standalone RF coils were obtained with a set of tuning capacitance values summarized in Table II. Besides, the capacitive balanced matching networks allowed an efficient 50-ohm matching and the corresponding values are reported in Table II.
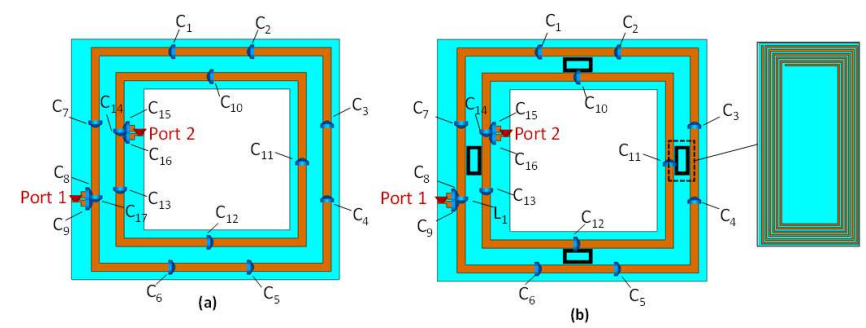

Fig. 3. Complete 3D numerical CAD of: (a) ${ }^{1} \mathrm{H}$ and ${ }^{23} \mathrm{Na}$ RF coils together without the SRs; and (b) ${ }^{1} \mathrm{H}$ and ${ }^{23} \mathrm{Na}$ RF coils together with the SRs in place. An inset of the used SR is inserted for clarity.

TABLE I

PHYSICAl PARAMETER DESCRIBING OUR TeSt CASE AT $298 \mathrm{MHz}$

\begin{tabular}{|c|c|c|c|c|c|c|}
\hline $\begin{array}{c}M_{{ }^{1} H^{23} \mathrm{Na}} \\
(\mathrm{nH})\end{array}$ & $\begin{array}{c}M_{{ }^{1} H S R} \\
(\mathrm{nH}) \\
\end{array}$ & $\begin{array}{c}M_{{ }^{23} \text { NaSR }} \\
(\mathrm{nH}) \\
\end{array}$ & $N$ & $\begin{array}{l}R_{S R} \\
(\Omega)\end{array}$ & $\begin{array}{c}L_{S R} \\
(\mathrm{nH})\end{array}$ & $\begin{array}{l}C_{S R} \\
(\mathrm{pF})\end{array}$ \\
\hline 117 & 24 & -24 & 4 & 10.8 & 748 & 0.37 \\
\hline
\end{tabular}

TABLE II

MATCHING AND TUNING CAPACITORS OF THE STANDALONE DT RF COIL

\begin{tabular}{ccc}
\hline \hline Capacitors & Function & Value \\
\hline$C_{1}, C_{2}, C_{3}, C_{4}, C_{5}, C_{6}, C_{7}$ & ${ }^{1} H$ tuning & $6.1 \mathrm{pF}$ \\
$C_{17}$ & ${ }^{1} H$ tuning & $27 \mathrm{pF}$ \\
$C_{8}, C_{9}$ & ${ }^{1} H$ matching & $60 \mathrm{pF}$ \\
$C_{10}, C_{11}, C_{12}, C_{13}$ & ${ }^{23} N a$ tuning & $82 \mathrm{pF}$ \\
$C_{14}$ & ${ }^{23} N a$ tuning & $20 \mathrm{pF}$ \\
$C_{15}, C_{16}$ & ${ }^{23} N a$ matching & $20 \mathrm{pF}$ \\
\hline \hline
\end{tabular}

TABLE III

MATCHING AND TUNING CAPACITORS OF THE DT RF COIL AFTER THE INSERTION OF 4 SRS

\begin{tabular}{ccc}
\multicolumn{3}{c}{ INSERTION OF 4 SRS } \\
\hline \hline$C_{1}, C_{2}, C_{3}, C_{4}, C_{5}, C_{6}, C_{7}$ & Function & Value \\
$L_{1}$ & ${ }^{1} H$ tuning & $5.8 \mathrm{pF}$ \\
$C_{8}, C_{9}$ & ${ }^{1} H$ tuning & $100 \mathrm{nH}$ \\
$C_{10}, C_{11}, C_{12}, C_{13}$ & ${ }^{1} \mathrm{H}$ matching & $8.8 \mathrm{pF}$ \\
$C_{14}$ & ${ }^{23} \mathrm{Na}$ tuning & $82 \mathrm{pF}$ \\
$C_{15}, C_{16}$ & ${ }^{23} \mathrm{Na}$ tuning & $82 \mathrm{pF}$ \\
\hline \hline
\end{tabular}

Figure 4 shows the reflection $\mathrm{S}$ parameters $\left(\mathrm{S}_{11}\right.$ and $\left.\mathrm{S}_{22}\right)$ of the standalone RF coils (solid lines) and the same coils placed together (dashed lines). When the RF coils are isolated, they present an excellent tuning and matching at the desired resonant frequencies. Then, the two RF coils were placed in a planar and concentric disposition to evaluate the degree of mutual coupling. Once arranged together, a noticeable upshift (about $25 \mathrm{MHz}$ ) of the ${ }^{1} \mathrm{H}$ resonant frequency is observed (Fig. 4 , dashed line) due to a strong mutual coupling, while the ${ }^{23} \mathrm{Na}$ resonant frequency is not affected. With the purpose of correcting the ${ }^{1} \mathrm{H}$ frequency shift, 4 SRs were inserted in between the RF coils, according to the design performed in Section III.A. As theoretically predicted in Section II.B, the insertion of the SRs caused a detuning and a mismatch of the ${ }^{1} \mathrm{H}$ loop. 


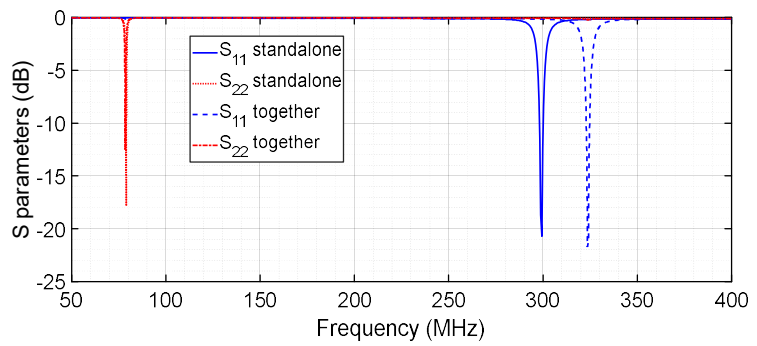

Fig. 4. Simulated $\mathrm{S}$ parameters $(\mathrm{dB})$ of the standalone ${ }^{1} \mathrm{H}\left(\mathrm{S}_{11}\right)$ and ${ }^{23} \mathrm{Na}\left(\mathrm{S}_{22}\right)$ RF coils (solid lines) versus the values when the two RF coils are placed together (dashed line) without the use of SRs.

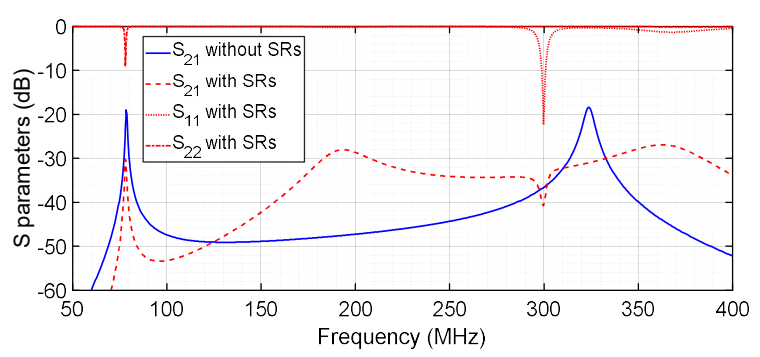

Fig. 5. Simulated $S$ parameters $(\mathrm{dB})$ of the two RF coils: $\mathrm{S}_{12}$ without SRs (dashdot line); $\mathrm{S}_{12}$ with $\mathrm{SRs}$ (solid line); $\mathrm{S}_{11}$ (dashed line, ${ }^{1} \mathrm{H}$ ) and $\mathrm{S}_{22}$ (dotted line, ${ }^{23} \mathrm{Na}$ ) with SRs.

Both effects were compensated by adjusting the lumped elements values (see Table III). At the end of the procedure, the two RF coils and the SRs worked together as predicted at the desired frequencies, with a drastic decoupling effect visible in the $S_{12}$ parameter, as shown in Fig. 5. It can be noticed that the $\mathrm{S}_{12}$ without the SRs shows a peak $(-18 \mathrm{~dB})$ at the upshifted ${ }^{1} \mathrm{H}$ resonant frequency of about $325 \mathrm{MHz}$, while, with the SRs, the restored resonant frequency for the ${ }^{1} \mathrm{H}$ loop is about $298 \mathrm{MHz}$, with a minimum $\mathrm{S}_{12}$ of $-40 \mathrm{~dB}$. Moreover, the results of Fig. 5 show that the $S_{12}$ at the ${ }^{23} \mathrm{Na}$ frequency improves from about -20 to $-30 \mathrm{~dB}$. These numerical fullwave results confirmed the efficacy of the novel decoupling method herein described.

\section{Distributed Magnetic Trap power handling}

Although being an attractive solution for decoupling purposes, spiral resonators can raise concerns about their power handling effective capability during a real case RF MRI high-power input signal. In particular, the small spacing between a single spiral and the MRI coils and their very tiny copper traces can be a potential source for electric arcing.

Thus, we performed full-wave simulations to obtain electric field distribution along the entire structure considering a highpower input signal at the coils' ports. By taking into account the typical amplitude required for a multi-nuclear sequence (especially for RF coils pre-scan calibration), we set the input signal as a $2 \mathrm{kWpp}$ continuous wave and we evaluated the electric field at a plane $20 \mu \mathrm{m}$ above the dielectric substrate. In this way, considering a $35 \mu \mathrm{m}$ thick copper trace, we obtained the field distribution also in between two adjacent spiral branches, which corresponds to the location with the smallest

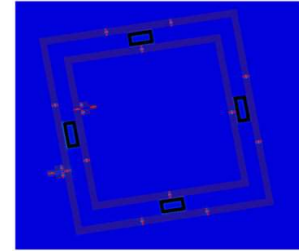

(a)

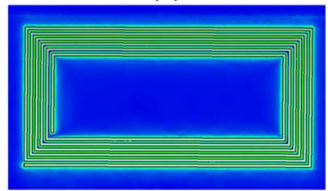

(b)

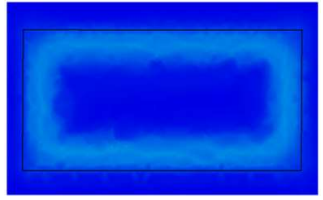

(c)

Fig. 6. (a) Electric field distribution in the complete MRI system at a plane 20 $\mu \mathrm{m}$ above the substrate: it can be seen that the peak values are in proximity of the spirals. (b) Particular of the field distribution on a spiral resonator, which corresponds to the highest field location. (c) Electric field inside the dielectric substrate. All the distributions show that the electric strength of the air is never overcome in the entire structure.

gap between conductors in the entire structure. As expected, the highest values of the field were located in correspondence of the spiral resonators (Fig. 6 (a)), dropping very quickly moving away from them; indeed, spirals' small dimensions and nested structure have a concentration effect on the field. However, taking as reference the experimental data provided in [39], [40] for the electric strength of the air between gaps in the order of $100 \mu \mathrm{m}$ (which is around $7 \mathrm{MV} / \mathrm{m}$ at $300 \mathrm{MHz}$ ), we found that no arcing risk is present in our design (Fig. 6 (b)). In addition, we evaluated also the field inside the dielectric slab (Fig. 6 (c)); considering that the dielectric has an electric strength much more pronounced than air, no problem was also present inside the slab.

Nonetheless, it may be worth noting that the electric arcing may become an issue when the feeding power approaches 4 $\mathrm{kW}$ (which is possible for particular MRI sequences); in that situation, the electric field gets closer to $7 \mathrm{MV} / \mathrm{m}$. A possible solution to alleviate the problem can consist in smoothing the $90^{\circ}$ bends of the spirals and reducing their tip angle.

\section{Sensitivity to errors evaluation}

Another important point to be analyzed consists in the sensitivity evaluation of the proposed design procedure against potential variations in the required parameters. Specifically, spiral resonators' self-impedance and their mutual coupling coefficients with MRI coils can be affected by errors since they are estimated by using a magneto-static approach.

We first calculated the S-parameters from the analytical model proposed in Section II by using the analytically estimated parameters and comparing them with full-wave simulations performed in CST (Fig. 7). Although a static 

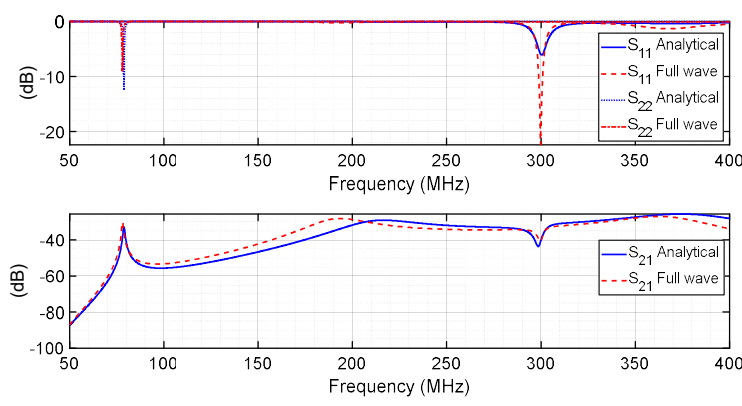

Fig. 7. Comparison between S-parameters obtained using the analytical model and full-wave simulations in presence of the spiral resonators: the magnetostatic approximation used to achieve filter design proved robust.
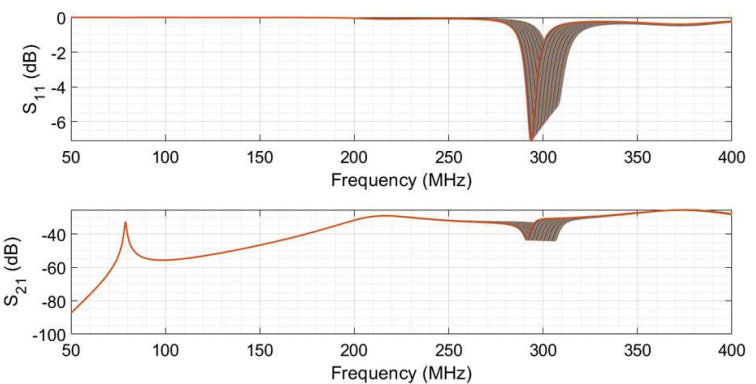

(a)
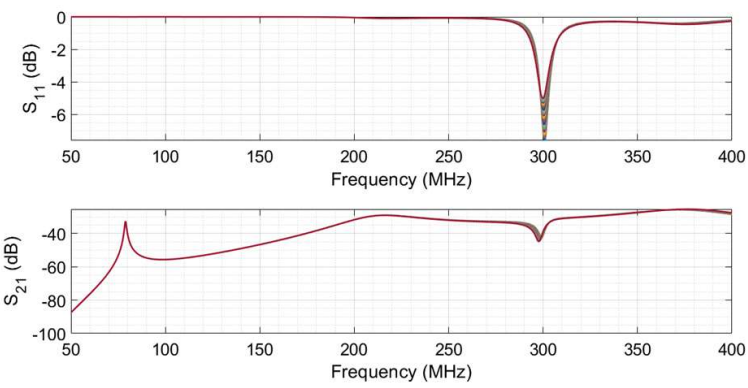

(b)

Fig. 8. $\mathrm{S}_{11}$ and $\mathrm{S}_{21}$ sensitivity evaluation with respect to error sources. (a) \pm 2.5 $\%$ variability on SRs' self impedance; (b) $\pm 10 \%$ variability on mutual coupling coefficients between SRs and MRI coils. In both cases, our design procedure is robust and can be applied without concerns.

approximation cannot fully describe all the phenomena happening in the system, it is sufficiently accurate in the unloaded condition to achieve a robust filter design, very close to the full-wave results.

After that, since we could not use full-wave simulations to evaluate possible errors on mutual coupling coefficients and SRs self-impedance estimations (they are estimated using analytical static models), we exploited again the circuital model. We imposed an error on the analytical parameters we originally used to design the filter (i.e., the results in Fig. 7) and we evaluated variations in S-parameters. We performed two analyses: one changing the values of the mutual coupling coefficients between SRs and RF coil loops, the other changing the SRs self-impedance (i.e., $L$ and $C$ ) (Fig. 8). We did not report the study performed on the ${ }^{23} \mathrm{Na}$ loop $\left(\mathrm{S}_{22}\right.$ parameter) because it was minimally affected by such variations, being significantly out of resonance around 300 $\mathrm{MHz}$. (a)

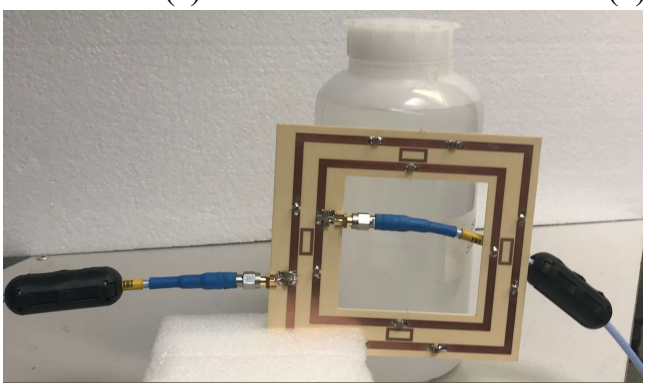

(c)

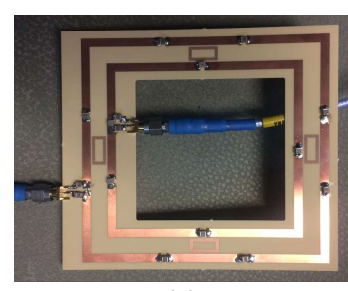

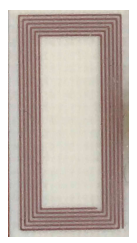

(b)
Fig. 9. ${ }^{1} \mathrm{H}$ and ${ }^{23} \mathrm{Na}$ RF coil prototypes in presence of the DMT filter: (a) unloaded system; (b) particular of a single spiral resonator; (c) complete system with a biological load (saline solution, $0.05 \mathrm{M}$ ).

We independently imposed a $\pm 2.5 \%$ of variability to the inductance and capacitance values of the resonators, and a $\pm 10 \%$ on the mutual coupling coefficients between SRs and MRI coils, in accordance to measurements uncertainties performed in [38]. As reported in Fig. 8, the design is overall robust against error sources; in particular, we obtained a \pm $2.26 \%$ frequency shift in the minimum of the $\mathrm{S}_{11}\left({ }^{1} \mathrm{H}\right.$ loop$)$ when the spirals' self-impedance is changing and $\mathrm{a} \pm 0.15 \%$ shift with the error on the mutual coupling coefficients. On the other hand, the frequency shift in the minimum of the $S_{21}$ is varying between $\pm 2.5 \%$ with SRs self-impedance uncertainties and $\pm 0.27 \%$ with mutual coefficients fluctuation.

Hence, we concluded that our design procedure is robust enough against the main sources of error and can be applied to real scenarios with confidence.

\section{E. Experimental verification}

We fabricated prototypes of the concentric RF coils without and with the SRs. The RF coils were tuned, matched and equipped with 50 -ohm coaxial connectors, evaluating both the unloaded configuration and the RF system in presence of a biological load (Fig. 9). As a biological load, we chose a cylindrical bottle of saline solution $(\mathrm{NaCl}, 0.05 \mathrm{M}), 18 \mathrm{~cm}$ height and with a $9 \mathrm{~cm}$ diameter. Its electric properties are similar to the human tissue at $300 \mathrm{MHz}\left(\varepsilon_{\mathrm{r}}=76, \sigma=0.56 \mathrm{~S} / \mathrm{m}\right)$.

We performed the measurements of the S-parameters at the Vector Network Analyzer (VNA) (E5071C ENA, Keysight). The measured S-parameters of the RF coils prototypes without and with SRs in the two loading conditions are shown in Fig. 10. As evident from the results, the presence of the SRs restores the correct resonant frequency for the ${ }^{1} \mathrm{H}$ RF coil and drastically reduces the mutual coupling with the ${ }^{23} \mathrm{Na}$ loop.

It must be pointed out that the slight differences in the experimental resonant frequencies with respect to the 


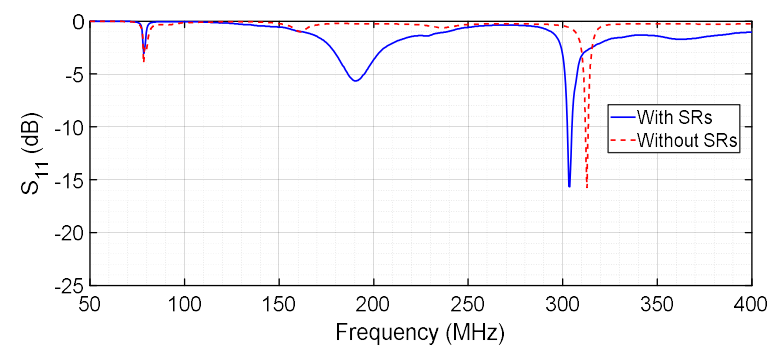

(a)

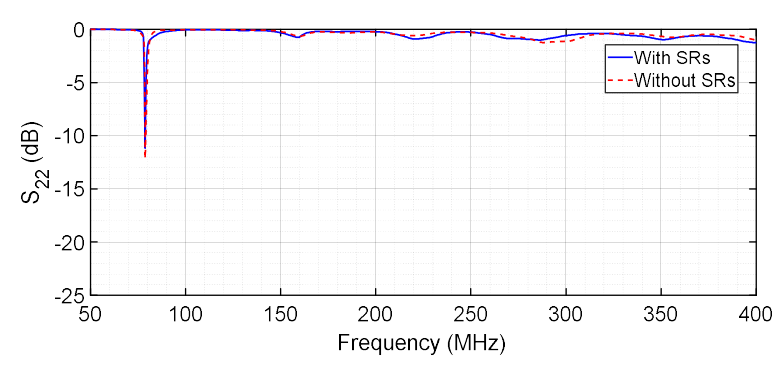

(b)

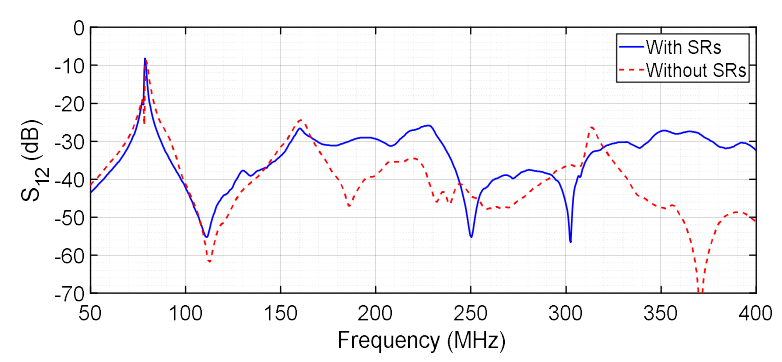

(c)

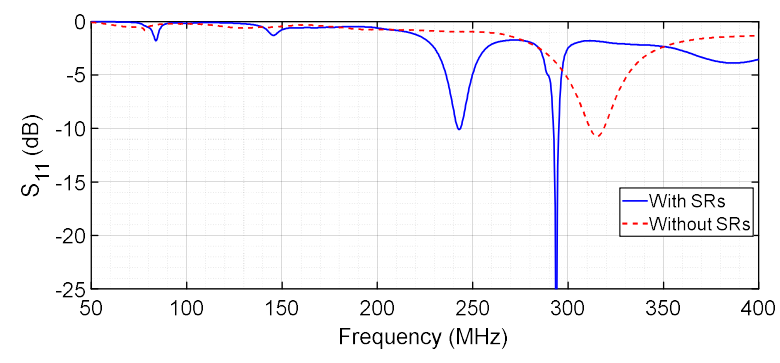

(d)

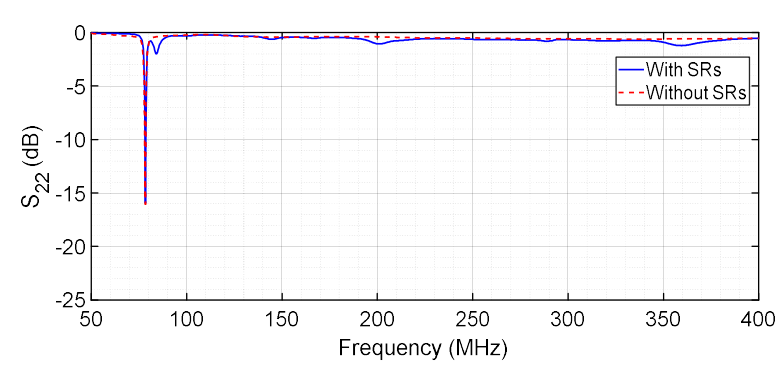

(e)

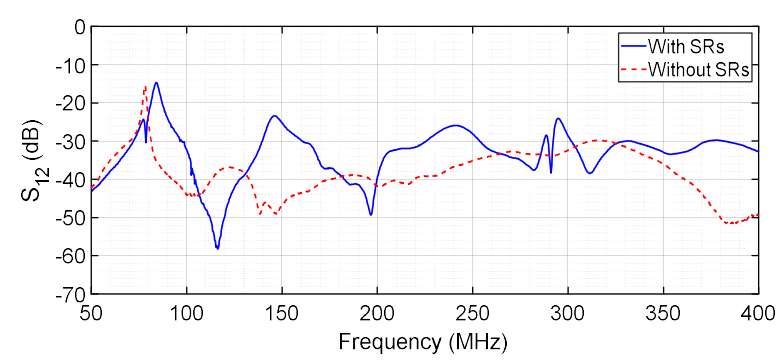

(f)

Fig. 10. Measured S parameters of the RF coil prototypes without (dashed line) and with (solid line) SRs. Left column: without biological load. Right column: with biological load. (a), (d) $\mathrm{S}_{11}$ parameter (dB) measured from the ${ }^{1} \mathrm{H}$ channel; (b), (e) $\mathrm{S}_{22}$ parameter (dB) measured from the ${ }^{23} \mathrm{Na}$ channel; (c), (f) $\mathrm{S}_{12}$ parameter (dB).

numerical simulations are in agreement with previous works [41], and are compatible with the tolerances of the lumped components and with imperfections of fabrication (e.g., etching, dielectric substrate variability, soldering procedure). The measurements variations were always within a $5 \%$ tolerance with respect to full-wave simulations, thus in good agreement with numerical design.

One possible concern about the SRs adoption is the negative effect on the efficiency of the RF coils due to additional resistive losses. In order to verify the impact of the SRs losses on the RF coils, we reported in Table IV the measured $\mathrm{Q}$ factor of the standalone ${ }^{1} \mathrm{H}$ and ${ }^{23} \mathrm{Na} \mathrm{RF}$ coils, without and with the SRs in place, obtained from the $S_{21}$ measurement $\left(\mathrm{Q}=f_{0} /\right.$ Bandwidth $\left.(-3 \mathrm{~dB})\right)$. We performed measurements in free space and with the biological phantom previously described. It can be seen that the measured $Q$ factors in unloaded condition showed a very small degradation for the ${ }^{23} \mathrm{Na}$ coil $(4 \%)$ and a moderate decrease for the ${ }^{1} \mathrm{H}$ coil (23\%). In the presence of the load, the ${ }^{1} \mathrm{H} \mathrm{Q}$-factor underwent an $18 \%$ decrease, while the ${ }^{23} \mathrm{Na}$ loop suffered from a $30 \%$ reduction. In general, the phantom presence is the cause for a TABLE IV

MEASURED Q-FACTORS OF ${ }^{1} \mathrm{H}$ AND ${ }^{23} \mathrm{NA}$ RF COILS WITHOUT AND WITH SPIRAL RESONATORS

\begin{tabular}{|c|c|c|c|c|}
\hline & $\begin{array}{c}\text { Without } \\
\text { SRs, no } \\
\text { load } \\
\end{array}$ & $\begin{array}{c}\text { With } \\
\text { SRs, no } \\
\text { load }\end{array}$ & $\begin{array}{l}\text { Without } \\
\text { SRs, } \\
\text { load } \\
\end{array}$ & $\begin{array}{l}\text { With } \\
\text { SRs, } \\
\text { load }\end{array}$ \\
\hline${ }^{1} H$ standalone $R F$ coil & 124 & 95 & 97 & 82 \\
\hline${ }^{23} \mathrm{Na}$ standalone $R F$ coil & 317 & 304 & 237 & 181 \\
\hline
\end{tabular}

significant decrease of the Q-factor, as it was expected. In general, these experimental results suggest that the sensitivity of the ${ }^{23} \mathrm{Na}$ coil, the most critical for in vivo applications because of the low natural ${ }^{23} \mathrm{Na}$ concentration in the tissues, remains significantly high even in presence of both the distributed trap and biological phantom. The larger losses measured in the ${ }^{1} \mathrm{H}$ RF coil are mostly due to the need of inserting the (lossy) inductor in series with the ${ }^{1} \mathrm{H}$ loop, in order to reach a good degree of matching/tuning. However, given the natural abundance of the ${ }^{1} \mathrm{H}$ signal, this is not so critical for the envisaged applications at $7 \mathrm{~T}$. Alternative 
matching/tuning methods requiring only high-quality capacitors can be employed in future designs of the ${ }^{1} \mathrm{H}$ coil in the presence of the SRs to improve the Q. Furthermore, it can be interesting to notice that the estimated losses when the spiral resonators are used as decoupling elements, are substantially aligned with what generally observed in the literature for traditional lumped trap circuits [12],[13]. Finally, it must be pointed out also that measurement errors can introduce some variability on these values.

\section{CONCLUSIONS}

A systematic analytical procedure for the design of distributed SRs, useful for the decoupling of MRI RF coils was introduced. We selected as test-case the design of a DT $\left({ }^{1} \mathrm{H} /{ }^{23} \mathrm{Na}\right) \mathrm{RF}$ coil configuration suitable for $7 \mathrm{~T}$ MRI, consisting of two concentric and coplanar loops. We have shown that the 4 SRs, printed on the same dielectric substrate of the RF coils, act as a distributed magnetic trap (DMT) circuit. The design of the whole decoupling system was performed through a fast and reliable fully analytical procedure, which is able to give a physical insight of the mutual coupling mechanism between the RF coils and SRs. Moreover, it is possible to choose the correct number, typology and position of the SRs, thus achieving a design close to the optimum. The refinement of the distributed SRs performance can be carried out through few targeted full-wave simulations. The feasibility of the proposed approach has been demonstrated by running full-wave simulations and experimental measurements on fabricated RF coil prototypes. Optimal decoupling (better than $-40 \mathrm{~dB}$ ) at the resonant frequency of the ${ }^{1} \mathrm{H}$ was achieved with 4 distributed SRs, placed symmetrically in between the ${ }^{1} \mathrm{H}$ and ${ }^{23} \mathrm{Na} \mathrm{RF}$ coils. Even in the presence of the SRs and of a realistic phantom, the quality factor of the ${ }^{23} \mathrm{Na}$ and ${ }^{1} \mathrm{H}$ coils remains significantly high to be suitable for practical scenarios.

The technology of printing the SRs over the same dielectric substrate of the RF coils results in a major advantage with respect to the traditional lumped elements trap circuit. Indeed, the flat design of the SRs allows a neat implementation without the need for extra space on top of the coplanar RF coils. Finally, although we selected as test-case the design of a DT RF coil configuration, the same analytical approach can be also extended to RF coil arrays suitable for parallel MRI.

\section{REFERENCES}

[1] P. B. Roemer et al., "The NMR phased-array", Magn. Reson. Med., vol. 16, pp. 192-225, 1990.

[2] D. K. Sodickson, W. J. Manning, "Simultaneous acquisition of spatial harmonics (SMASH): fast imaging with radiofrequency coil arrays", Magn. Reson. Med., vol. 38, pp. 591-603, 1997.

[3] K. P. Pruessmann et al., "SENSE: sensitivity encoding for fast MRI", Magn. Reson. Med., vol. 42, pp. 952-962, 1999.

[4] M. Weiger, K. P. Pruessmann, P. Boesiger, "Cardiac real-time imaging using SENSE. SENSitivity Encoding scheme”, Magn. Reson. Med., vol. 43, pp. 177-184, 2000.

[5] J. A. de Zwart et al., "Design of a SENSE-optimized high-sensitivity MRI receive coil for brain imaging", Magn. Reson. Med., vol. 47, pp 1218-1227, 2002
[6] J. A. de Zwart et al., "Signal-to-noise ratio and parallel imaging performance of a 16-channel receive-only brain coil array at 3.0 Tesla", Magn. Reson. Med., vol. 51, pp. 22-26, 2004.

[7] B. Keil, L. L. Wald, "Massively parallel MRI detector arrays", J. Magn. Reson., vol. 229, pp. 75-89, 2013.

[8] C. H. Moon et al., "Quantitative 23Na MRI of human knee cartilage using dual-tuned $1 \mathrm{H} / 23 \mathrm{Na}$ transceiver array radiofrequency coil at 7 Tesla", J. Magn. Reson. Imaging, vol. 38, pp. 1063-1072, 2013.

[9] A. Abuelhaija, S. Orzada, K. Solbach, "Parasitic Element Based Decoupling of 7 Tesla MRI Coil Array", Antennas \& Propagation Conference ( $L A P C)$, Loughborough, 2015.

[10] G. Adriany et al., "A 32-channel lattice transmission line array for parallel transmit and receive MRI at 7 Tesla", Magn. Reson. Med., vol. 63, pp. 1478-1485, 2010.

[11] A. M. Maunder et al., "Stray capacitance between MRI coil elements: Models and application to array decoupling", IEEE Trans. Microw. Theory Techn., vol. 61, pp. 4667-4677, 2013.

[12] M. Alecci et al., "Pratical design of a 4 Tesla double-tuned RF surface coil for interleaved $1 \mathrm{H}$ and $23 \mathrm{Na}$ MRI of rat brain", J. Magn. Reson., vol. 181, pp. 203-211, 2006.

[13] M. Meyerspeer et al., "An Improved Trap Design for Decoupling Multinuclear RF Coils”, Magn. Reson. Med., vol. 72, pp. 584-590, 2014.

[14] J. V. Rispoli et al., "Trap design and construction for high-power multinuclear magnetic resonance experiments", Concepts Magn. Reson. Part B, vol. 46B, pp. 162-168, 2016.

[15] U. Zanovello et al., "Experimental setup to compare measurements and numerical simulations in Magnetic Resonance Imaging RF Dosimetry”, IEEE Trans. Instrum. Meas., Vol. 66, pp. 1208-1216, 2017.

[16] B. Park et al., "Faraday shields within a solenoidal coil to reduce sample heating: Numerical comparison of designs and experimental verification", J. Magn. Reson., vol. 202, pp. 72-77, 2010.

[17] G. Adriany, R. Gruetter, "A Half-Volume Coil for Efficient Proton Decoupling in Humans at 4 Tesla", J. Magn. Reson., vol. 125, pp. 178184, 1997.

[18] Y. Li et al., "ICE decoupling technique for RF coil array designs", Med. Phys., vol. 38, pp. 4086-4093, 2011.

[19] X. Yan et al., "Magnetic wall decoupling method for monopole coil array in ultrahigh field MRI: a feasibility test", Quant. Imaging Med. Surg. vol. 4, pp. 79-86, 2014.

[20] E. Georget et al., "Stacked magnetic resonators for MRI RF coils decoupling”, J. Magn. Reson., vol. 275, p. 11-18, 2017.

[21] N.I. Avdievich, J.W. Pan, H.P. Hetherington, "Resonant inductive decoupling (RID) for transceiver arrays to compensate for both reactive and resistive components of the mutual impedance", NMR Biomed., vol. 26, pp. 1547-1554, 2013.

[22] B. Wu et al., "Interconnecting L/C components for decoupling and its application to low-field open MRI array", Concepts Magn. Reson. Part $B$, vol. 31B, pp. 116-126, 2007.

[23] C. Von Morze et al., "An eight-channel, nonoverlapping phased array coil with capacitive decoupling for parallel MRI at 3 T", Concepts Magn. Reson. Part B, vol. 31B, pp. 37-43, 2007.

[24] X. Zhang, A, Webb, "Design of a capacitively decoupled transmit/receive NMR phased array for high field microscopy at 14.1 T”, J. Magn. Reson., vol. 170, pp. 149-155, 2004.

[25] K. M. Gilbert et al., "A radiofrequency coil to facilitate $\mathrm{B}_{1}{ }^{+}$shimming and parallel imaging acceleration in three dimensions at $7 \mathrm{~T}$ ", NMR Biomed., vol. 24, pp. 815-823, 2011.

[26] I. R. O. Connell et al., "MRI RF array decoupling method with magnetic wall distributed filters," IEEE Trans. Med. Imag., vol. 34, pp. 825-835, 2015.

[27] I. R. O. Connell et al., "Design of a parallel transmit head coil at 7T with magnetic wall distributed filters", IEEE Trans. Med. Imag., vol. 34, pp. 836-845, 2015.

[28] I. R. O. Connell, R. S. Menon, "General coupling matrix synthesis for decoupling MRI RF arrays", IEEE Trans. Med. Imag., vol. 35, pp. 22292242, 2016.

[29] F. Bilotti, A. Toscano, L.Vegni, "Design of spiral and multiple split-ring resonators for the realization of miniaturized metamaterial samples, IEEE Trans. Antennas Propag., vol. 55, pp. 2258-2267, 2007. 
[30] F. Bilotti et al., "Equivalent-circuit models for the design of metamaterials based on artificial magnetic inclusions", IEEE Trans. Microw. Theory Techn., vol. 55, pp. 2865-2873, 2007.

[31] K. B. Alici et al., "Optimization and tunability of deep subwavelength resonators for metamaterial applications: complete enhanced transmission through a subwavelength aperture", Optics Express, vol. 17, pp. 5933-5943, 2009.

[32] J. D. Kaggie et al., "7 T Sodium/Proton Knee Imaging: First Results", ISMRM UHF Symposium, Heidelberg, Germany, p.21, 2016.

[33] N. Fontana et al., "Distributed Trap FSS Filter for Dual Tuned RF MRI Coil Decoupling at 7.0T", IEEE International Conference on Electromagnetics in Advanced Applications (ICEAA), 2017.

[34] N. Fontana et al., "Decoupling of Dual-Tuned MRI Coils by Using Distributed Magnetic Traps", IEEE $12^{\text {th }}$ European Conference on Antennas and Propagation (EuCAP), 2018.

[35] D. Brizi et al., "On the Optimization of Distributed Magnetic Traps in MRI Coils Decoupling", IEEE International Symposium on Antennas and Propagation \& USNC/URSI National Radio Science Meeting, 2018.

[36] Y. Cheng, Y. Shu, "A new analytical calculation of the mutual inductance of the coaxial spiral rectangular coils", IEEE Trans. Magn., vol. 50, no. 4, 7026806, 2014.

[37] F. Costa, A. Monorchio, G. Manara, "An overview of equivalent circuit modeling techniques of Frequency Selective Surfaces and metasurfaces", ACES Journal, vol. 29, 2014.

[38] D. Brizi et al., "Accurate Extraction of Equivalent Circuit Parameters of Spiral Resonators for the Design of Metamaterials", IEEE Trans. Microw. Theory Techn., vol. 67, no. 2, pp. 626-633, Feb. 2019.

[39] J. A. Pim, "Electrical Breakdown Strength of Air at Ultra HighFrequencies", Nature, vol. 161, no. 4096, p. 683, 1948.

[40] J. A. Pim, "The electrical breakdown strength of air at ultra-high frequencies", Proceedings of the IEE-Part III: Radio and Communication Engineering, vol. 96, no. 40, pp. 117-129, 1949.

[41] R. Stara et al., "Validation of numerical approaches for electromagnetic characterization of magnetic resonance radiofrequency coils", Prog. Electromagn. Res. M., vol. 29, p. 121-136, 2013. 